\title{
Aspectos atuais no diagnóstico do edema macular diabético
}

\section{Recent aspects on diagnosis of diabetic macular edema}

Mário Martins dos Santos Motta ${ }^{1}$, Jacqueline Coblentz ${ }^{2}$, Laura Gomes Nunes de Melo ${ }^{3}$

\section{ResUMo}

O diagnóstico do edema macular diabético deve ser feito precocemente, a fim de evitar perda visual significativa. Para tal, contamos com exames complementares cada vez mais desenvolvidos e específicos. Os autores fazem uma revisão dos principais métodos existentes para diagnosticar a retinopatia diabética.

Descritores: Retinopatia diabética/diagnóstico; Edema macular/diagnóstico; Angiofluoresceinografia;Tomografia de coerência óptica

\footnotetext{
'Livre-docente, Professor Adjunto da Universidade Federal do Estado do Rio de Janeiro - UNIRIO - Rio de Janeiro (RJ), Brasil; Responsável pelo Setor de Retina e Vítreo do Hospital dos Servidores do Estado - HSE - Rio de Janeiro (RJ), Brasil; Professor Titular da Faculdade de Medicina de Teresópolis - UNIFESO - Rio de Janeiro (RJ), Brasil;

${ }^{2}$ Médica oftalmologista da Universidade Federal do Estado do Rio de Janeiro UNIRIO - Rio de Janeiro (RJ), Brasil;

${ }^{3}$ Residente do Hospital dos Servidores do Estado - HSE - Rio de Janeiro (RJ), Brasil.
} 


\section{INTRODUÇÃO}

A diminuição da visão central, especialmente para perto, é o principal sintoma nas doenças maculares. A escala de "BaileyLovie", adotada pelo ETDRS, é o padrão de avaliação da visão central em publicações científicas ${ }^{(1)}$. O edema macular diabético (EMD) só causa alterações visuais quando há acometimento da fóvea ou da retina imediatamente adjacente ${ }^{(2,3)}$.

O EMD pode acompanhar-se de metamorfopsia e/ou micropsia, que são bem caracterizadas pelo teste com a grade de Amsler ${ }^{(3)}$.

Para diagnóstico clínico do EMD, examina-se cuidadosamente a retina do paciente sob midríase, com oftalmoscopia direta, biomicroscopia de fundo ou oftalmoscopia indireta ${ }^{(3)}$.

As alterações da retinopatia diabética (RD), descritas a seguir, iniciam-se na área temporal à mácula, provavelmente por ser uma área crítica na circulação retiniana onde ocorre o fechamento dos capilares retinianos adjacentes à área foveal avascular ${ }^{(4)}$ :

- Microaneurismas: resultam da oclusão capilar. São vistos à fundoscopia como pequenos pontos vermelhos, com bordos bem definidos. Ocorrem devido à proliferação de células endoteliais a partir do leito venoso dos capilares e correspondem à primeira alteração oftalmoscópica da RD;

- Manchas algodonosas (exsudatos moles): são observadas como manchas brancas superficiais, bem delimitadas de bordos irregulares, que tendem a desaparecer em semanas ou meses. Resultam da isquemia microvascular na camada de fibras nervosas da retina, causando infarto e destruição dos axônios. Histologicamente correspondem a acúmulo de organelas, pela alteração no transporte axoplasmático das fibras acometidas;

- Exsudatos duros: são depósitos de lipídios e/ou lipoproteínas e indicam aumento da permeabilidade vascular retiniana. São vistos como pontos amarelos bem circunscritos e profundos em relação aos vasos da retina;

- Hemorragias: decorrem de lesão da parede vascular. Quando puntiformes são indistinguíveis dos microaneurismas sem o auxílio da angiografia fluoresceínica. Podem ocorrer também na camada de fibras nervosas, assemelhando-se à "chama de vela", ou ainda originar-se do plexo capilar profundo, com aspecto "em borrão".

- Edema macular: é definido pela presença de qualquer espessamento retiniano ou exsudato duro dentro da área correspondente a um diâmetro papilar $(1500 \mathrm{~mm})$, a partir do centro da fóvea ${ }^{(2,3)}$. Define-se edema macular clinicamente significativo pela presença de um ou mais dos seguintes achados, de acordo com o ETDRS ${ }^{(1)}$ :

$>$ Edema retiniano dentro de $500 \mathrm{~mm}$ de distância a partir do centro da fóvea;

$>$ Exsudato duro dentro de $500 \mathrm{~mm}$ a partir do centro da fóvea, se associado com espessamento retiniano adjacente (que pode estar além dos $500 \mathrm{~mm}$ );

$>$ Edema retiniano do tamanho de um diâmetro papilar $(1500 \mathrm{~mm})$ ou maior, e qualquer parte deste esteja dentro do diâmetro de um disco papilar, a partir do centro da fóvea.

\section{Exames complementares Angiografia fluoresceínica}

A fluoresceína absorve energia luminosa entre 465-490 nm (luz azul), e emite fluorescência entre 520$530 \mathrm{~nm}$ (luz verde), enquanto for mantida a iluminação de excitação. Após aproximadamente 8 a 12 segundos da injeção, atinge o olho e preenche artérias e, logo depois, as veias ${ }^{(3)}$.

Pode haver impregnação da pele, esclera e membranas mucosas, e a coloração amarelada que persiste durante 2 a 6 horas. $O$ endotélio vascular da retina e do cérebro, bem como o EPR, são impermeáveis ao corante $^{(3,6)}$.

A fluoresceína sódica é uma substância de baixa toxicidade, podendo ser utilizada em gestantes e neonatos. Algumas reações adversas ao corante são náuseas, vômitos, resposta vasovagal, urticária e síncope. É metabolizada pelo fígado e eliminada pelos rins em 24 horas. Pacientes com insuficiência hepática ou renal não têm contraindicação para a realização do exame, apenas demoram mais a eliminar o corante. É contra-in-dicada em pacientes alérgicos a corante amarelo ${ }^{(7)}$.

No olho normal, a angiografia fluoresceínica apresenta as seguintes fases ${ }^{(3)}$ :

- Fase coroidiana: fluoresceína difusa no fundo, devido às fenestrações existentes nos capilares coroidais permitindo extravasamento de corante. $\mathrm{O}$ enchimento é súbito e irregular, chamado de flush coroidiano;

- Fase arterial: presença do corante nos ramos arteriolares retinianos. A fluoresceína fica confinada no espaço intravascular devido à presença da barreira hematorretiniana interna, que corresponde às junções 


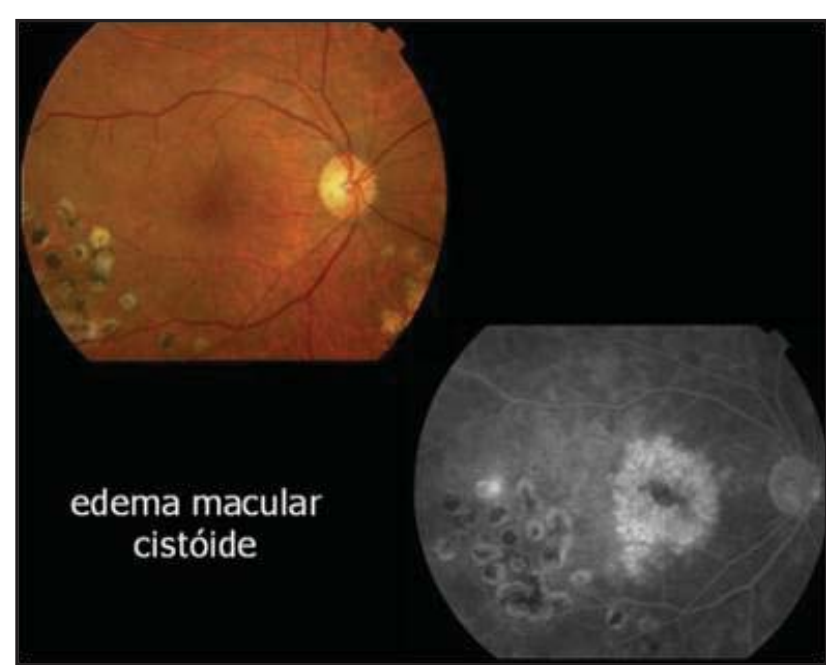

Figura 1: Angiografia fluoresceínica mostrando áreas de hiperfluorescência na região macular

apertadas entre as células endoteliais dos vasos retinianos;

- Fase venosa precoce (fase artériovenosa ou capilar): disposição laminar do contraste nos ramos venosos e perfusão total dos capilares;

- Fase venosa propriamente dita: veias totalmente preenchidas e arteríolas já esvaziando;

- Fase venosa tardia: ausência de contraste nas arteríolas. Nesta fase a fluorescência de fundo atinge seu máximo;

- Fase tardia ou tecidual: ausência de contraste nos ramos vasculares retinianos, com impregnação da esclera e do nervo óptico.

\section{Alterações angiográficas na RD Hiperfluorescência}

É o aumento da fluorescência em determinada área em relação às áreas circunvizinhas. Existem duas barreiras naturais à difusão do contraste: a barreira hematorretiniana interna, presente no endotélio das paredes dos vasos retinianos, e a barreira hemato-retiniana externa, formada pelas junções apertadas entre as células do epitélio pigmentário da retina, que impedem passagem de substâncias da circulação coriocapilar para o espaço sub-retiniano e retina ${ }^{(3)}$.

Na RD, a hiperfluorescência ocorre pelo escape do corante para o espaço intersticial, a partir da rede vascular retiniana, devido ao comprometimento da barreira interna (endotélio vascular) ${ }^{(3)}$.

A mácula normal é afluorescente devido à ausência de vasos nesta área e pela presença de um pigmento amarelado, a xantofila, encontrada na camada

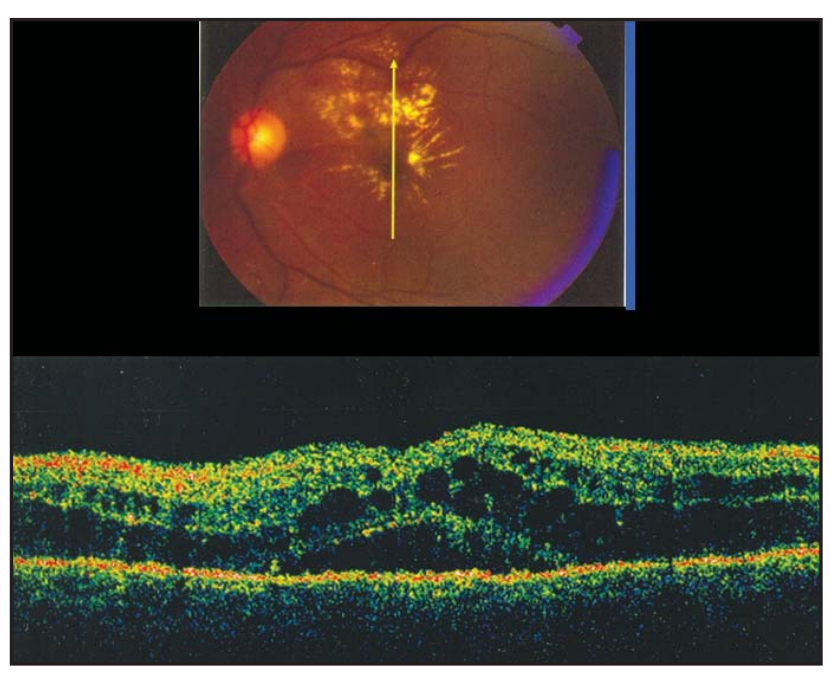

Figura 2: Tomografia de coerência óptica mostrando formação de espaços cistóides intrarretinianos e presença de líquido sub-retiniano

plexiforme externa da retina, especialmente na área foveal. Além disso, as células do epitélio pigmentário nesta região são mais altas e pigmentadas, dificultando a transmissão de fluorescência coroidiana ${ }^{(6)}$.

Os microaneurismas são as primeiras alterações anatômicas a aparecer nos vasos retinianos em decorrência do diabetes, sendo pontos hiperfluorescentes a partir da fase capilar. O diagnóstico é precoce devido ao menor peso molecular da fluoresceína em relação à hemácia, podendo penetrar no microaneurisma ainda em formação, permitindo sua identificação antes da fundoscopia. Os neovasos são sempre hiperfluorescentes e quanto maior sua atividade, maior a hiperfluorescência ${ }^{(3)}$.

O edema na região macular é observado devido à exsudação serosa a partir da rede capilar macular e dos microaneurismas. Quando se acumula na camada plexiforme externa da retina por entre as fibras de Henle, há formação de espaços pseudocísticos com aspecto petalóide ou em rosácea, denominando-se edema cistóide da mácula (Figura 1). O edema pode ser mais difuso, sem apresentar o aspecto petalóide ${ }^{(6)}$.

\section{Hipofluorescência:}

Pode ocorrer por dois mecanismos: impedimento da visibilidade da fluoresceína de fundo por interposição de uma substância opaca causando bloqueio ou efeito máscara (ex: sangue na hemorragia pré-retiniana; proliferação pigmentária nos focos de coriorretinite) e por não-perfusão nas áreas isquêmicas ${ }^{(3)}$.

Os microaneurismas obliterados ficam hipofluorescentes durante todo o exame, assemelhando-se com as micro-hemorragias retinianas, o que difi- 
culta o diagnóstico diferencial mesmo com a angiografia ${ }^{(3)}$.

Nas oclusões capilares, surgem as manchas algodonosas que aparecem como zona de não-perfusão capilar retiniana, rodeada por microaneurismas e dilatações capilares. As manchas algodonosas correspondem a edema isquêmico das fibras nervosas, sendo hipofluorescentes ${ }^{(3,6)}$.

As dilatações em rosário da parede venosa e as alças venosas geralmente se formam próximo de grandes áreas isquêmicas, e aparecem na fase tardia (venosa) do exame. Não devem ser confundidas com neovasos ${ }^{(3)}$.

Os exsudatos duros e as hemorragias são mais bem observados na fotografia padrão, antes da injeção da fluoresceína. O extravasamento lipídico, quando ocorre na mácula, revela permeabilidade vascular anormal, a partir de microaneurismas ou capilares alterados. Isso permite concluir que há edema na região macular, que aparece na angiografia com áreas de hiperfluorescência tardia ${ }^{(3,6)}$.

Tomografia de coerência óptica - OCT (Optical Coherence Tomography)

A tomografia de coerência óptica (OCT) é um exame de alta resolução, não-invasivo e de não-contato, que usa luz com comprimento de onda próximo ao infravermelho, para produzir imagens seccionais da retina, em sentido sagital. Fornece imagem quantitativa e qualitativa da retina, assemelhando-se a um corte histológico e que permite avaliar a espessura retiniana e a camada de fibras nervosas ${ }^{(8.9)}$.

O OCT faz a análise entre a luz refletida das diferentes profundidades retinianas e as transforma em imagens com escala de cores falsas, de acordo com a maior ou menor reflectividade de cada estrutura ${ }^{(8)}$.

\section{Tomografia de coerência óptica e EMD}

Estudos recentes vêm demonstrando a superioridade do OCT no diagnóstico precoce do EMD, como sendo mais sensível do que o exame com biomicroscopia ou angiografia fluoresceínica na análise quantitativa do espessamento macular ${ }^{(6,10,11)}$.

Os padrões de espessamento da retina incluem alargamento isolado e difuso das camadas externas da retina, formação de espaços cistóides intraretinianos e a presença de líquido sub-retiniano ${ }^{(11,12)}$ (Figura 2). Admite-se como ponto de corte da espessura retiniana, medido pelo OCT, o valor de $180 \mathrm{~mm}$ para a espessura foveal. Este dado determinaria a presença ou ausência do EMD, podendo ser útil para detecção precoce do edema macular $(93 \%$ de sensi- bilidade e $75 \%$ de especificidade), indicando a necessidade de acompanhamento mais rigoroso desses pacientes ${ }^{(10)}$.

O OCT contribuiu para o melhor conhecimento da anatomia do EMD fornecendo o mapeamento da espessura macular, onde as cores mais claras correspondem a áreas mais espessas e as cores mais escuras, a regiões menos espessas. Este mapeamento pode auxiliar inclusive no tratamento da maculopatia diabética. Realizando-se o OCT antes e após a fotocoagulação com laser, é possível identificar áreas espessas que devem ser priorizadas, assim como a resolução ou não do edema após o tratamento ${ }^{(11)}$.

Além disso, o uso cada vez mais frequente do OCT para diagnosticar edema macular diabético tornou possível a detecção mais precoce, com instituição de tratamento em fase mais favorável quanto ao prognóstico visual. Por outro lado, com o diagnóstico baseado apenas no exame clínico, alguns pacientes foram submetidos à fotocoagulação com laser sem necessidade, já que o edema em realidade não existia, ocasionando alterações no campo visual paracentral por iatrogenia ${ }^{(11}$.

Outra contribuição do OCT foi a possibilidade de avaliação da profundidade dos exsudatos duros, que em geral encontram-se no interstício da retina. A presença de exsudatos no espaço sub-retiniano está associada a mau prognóstico visual ${ }^{(10,12)}$.

\section{Conclusão}

Com a utilização destes exames, hoje em dia é possível quantificar com maior acurácia o edema macular, principalmente nos casos em que a biomicroscopia de mácula não é conclusiva. Além disso, são importantes para determinar qual o melhor tipo de tratamento a ser empregado e avaliar a resposta terapêutica.

\section{Abstract}

Diabetic macular edema must be assessed as early as possible, to avoid significant vision loss. To achieve this goal, there are many specific diagnostic tests that can be performed. The authors review the most common methods in diagnosing diabetic retinopathy.

Keywords: Diabetic retinopathy/diagnosis; Macular edema/diagnosis; Fluorescein angiography; Tomography, optic coherence 


\section{RefERÊNCIAS}

1. Motta MMS. Doenças maculares adquiridas In: Kanski JJ. Oftalmologia clínica: uma abordagem sistemática. 5a ed. Rio de Janeiro: Elsevier; 2004. cap. 13. p. 390-405.

2. Ministério da Saúde. Indicadores de morbidade e fatores de risco: D.10 Taxa de prevalência de diabete melito [Internet] [citado 2007 Dez 12]. Disponível em: http:// tabnet.datasus.gov.br/cgi/idb2006/d10.htm

3. Dantés D, Siqueira RC. Angiografia da retina - fluoresceína e indocianina verde. Rio de Janeiro: Editora Revinter; 2004

4. Antcliff RJ, Marshall J. The pathogenesis of edema in diabetic maculopathy. Semin Ophthalmol. 1999;14(4):223-32.

5. Photocoagulation for diabetic macular edema. Early Treatment Diabetic Retinopathy Study report number 1.Early Treatment Diabetic Retinopathy Study research group. Arch Ophthalmol. 1985;103(12):1796-806.

6. Reivellese M, George A, Sulkes D, Reichel E, Puliafito C. Optical coherence tomography after laser photocoagulation for clinically significant macular edema. Ophthalmic Surg Lasers. 2002;31(3):192-7.

7. The effect of intensive treatment of diabetes on the development and progression of long-term complications in insulin-dependent diabetes mellitus. The Diabetes Control and Complications Trial Research Group. N Engl J Med. 1993;329(14):977-86
8. Schaudig U. [Optical coherence tomography]. Ophthalmologe. 2001;98(1):26-34. Review. German.

9. Sánchez-Tocino H, Alvarez-Vidal A, Maldonado MJ, Moreno-Montañés J, García-Layana A. Retinal thickness study with optical coherence tomography in patients with diabetes. Invest Ophthalmol Vis Sci. 2002;43(5):1588-94.

10. Browing DJ, McOwen MD, Bowen RM Jr, O'Marah TL. Comparison of the clinical diagnosis of diabetic macular edema with diagnosis by optical coherence tomography. Ophthalmology. 2004;111(4):712-5.

11. Otani T, Kishi S, Maruyama Y: Patterns of diabetic macular edema with optical coherence tomography. Am J Ophthalmol. 1999;127(6):688-93.

12. Moorman CM, Hamilton AM. Clinical applications of the MicroPulse diode laser. Eye. 1999;13(Pt 2):145-50.

\section{ENDEREÇO PARA CORRESPONDÊNCIA: \\ Jacqueline Coblentz}

Rua Visconde de Silva, $n^{\circ} 52$ - Ap. 703

CEP 22271-090 - Rio de Janeiro - RJ - Brasil

Tel/fax: 2286-5744/2266-3710

e-mail: j_coblentz@yahoo.com 